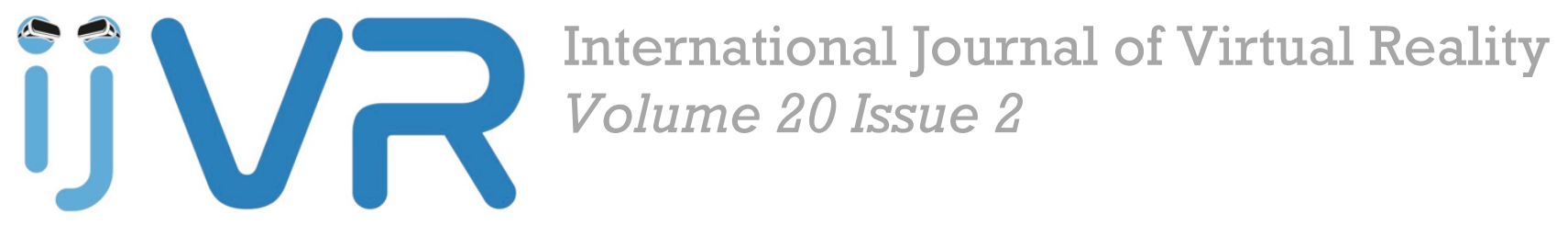

Araiza-Alba P., Keane T., Chen W. S., \& Kaufman J. (2020).

Children's memory of a story experienced with virtual reality versus traditional media technology, 20(2), 55-69.

https://doi.org/10.20870/IJVR.2020.20.2.3151

CC BY 4.0 license

Received: 3/2020

Published: $12 / 2020$ 


\title{
Children's memory of a story experienced with virtual reality versus traditional media technology
}

\author{
Paola Araiza-Alba, Therese Keane, Won Sun Chen, Jordy Kaufman \\ Swinburne University of Technology, PO Box 218, Hawthorn, Victoria, Australia
}

Corresponding author: Paola Araiza-Alba, paraiza@swin.edu.au, +61392148822

Keywords: Virtual Reality - Children - Memory - Emotions

\begin{abstract}
The purpose of this study is to evaluate the impact of immersive virtual reality (IVR) on children's memory of a story. Although long-term memory is essential for learning and IVR has been suggested as a tool to enhance learning, existing work has not examined how IVR presentations, compared with other types of storytelling, facilitates or interferes with children's memory formation. Here, we present data from a study of 70 6- to 7 year-old children randomly assigned to experience a story in one of three modalities: IVR, video, or picture book. We assessed the children's story recall and their ability to identify the protagonist's emotions. Results showed that, overall, children in the IVR condition performed better in the memory recall task than the children in the video and book conditions. The most pronounced difference in memory performance was between the IVR and book conditions. Looking at age groups separately, 6-year-olds' recall was significantly better in the IVR condition than in the video condition, while 7-year-olds performed similarly in both digital-story conditions. We found no effect of condition on children's attribution of emotions to the story's protagonist. We conclude that IVR can enhance children's ability to learn story content in certain situations.
\end{abstract}

\section{Introduction}

With the rapid growth of technological advances, such as high-speed computing, high-resolution graphics, and interface devices, immersive virtual reality technology (IVR) has become not only an exciting entertainment technology but one with many practical applications (Araiza-Alba et al., 2020a; Ke \& Im, 2013; Wilson et al., 2015) in areas including military or medical training (Bhagat et al., 2016; Jensen \& Konradsen, 2018), medical rehabilitation (Bryant et al., 2020), pain distraction (Kipping et al., 2012), neuropsychology assessment and tourism (Loureiro et al., 2019).

From a very young age, children are inclined to interact with technology (Hirsh-Pasek et al., 2015; Huber et al., 2018). Research on children's experiences with emerging technologies shows that depending on the implementation of the interaction, these experiences can have both learning (e.g., Huber et al., 2016, 2020; J. Tarasuik et al., 2017; Wang et al., 2021) and social benefits (e.g., Tarasuik et al. (2011, 2013); Tarasuik \& Kaufman (2017)). IVR likewise has the potential to be used as a pedagogical tool and as an immersive space for student learning, providing the learners with an authentic context in which they can develop their scope of learning, visualize situations and concepts that are not possible to display on other mediums, and obtain more meaningful knowledge (Chen, 2016; Huang et al., 2010; Ibáñez et al., 2011). IVR can provide realism to the learning experience, allowing more practical learning and understanding, and thereby resulting in improved levels of cognitive functioning (Papanastasiou et al., 2018).

Furthermore, virtual reality (VR) environments may have strong motivational impact on children's learning due to the immersive nature and sense of presence inherent in applications of this technology (Bricken, 1991; Maria et al., 1999; Slater \& Wilbur, 1997; Winn, 2010). Additionally, sense of presence and immersion are 
frequently associated with greater levels of involvement and more intense emotions, aspects that are associated with improved learning outcomes (Dibbets \& Schulte-Ostermann, 2015; Jablon \& Wilkinson, 2006). Hence, the higher the sense of presence and emotional involvement during the experience, the greater likelihood the participants will recall, the content presented to them (Mantovani \& Castelnuovo, 2003).

A child's ability to recall, recognize, or reproduce previously presented information has been shown to improve with increasing age (Gathercole, 1998). Adult memory performance is achieved around the age of 9-10 years or during adolescence (León et al., 2014). VR has been linked with the ability to aid recall. For example, Krokos, Plaisant, and Varshney (2019), examined memory recall of faces seen in via VR or on a computer monitor. Forty participants were instructed to memorize and then recall faces displayed in different locations within a virtual palace. The results showed that the participants completing the experiment via IVR had signficantly greater memory performance compared to the participants that used the desktop application. This finding is consistent with a number of other experiments with adult participants that demonstrate benefits of IVR in visual search, detection of information, and memory recall (Harman et al., 2017; Krokos et al., 2019; Mania et al., 2003; Pausch et al., 1997). Taken together, these results suggest that IVR experiences have the potential to boost learning of content.

Furthermore, people learn more and retain more when information is presented multiple times and through multiple channels - a phenomenon described as the "multimedia effect" (Mayer, 2003). VR systems can present information using a range of delivery mechanisms, through multiple channels or senses, and multiple times. It is auditory, visual, and more importantly, contains nonverbal auditory stimulus that traditional education methods do not have. Auditory cues, such as the sound of a door closing or the sound of bonds breaking or molecules reacting in a chemistry class, provide realism to the learning experience, allowing more practical learning and understanding (Bell et al., 1995).

Moreover, the emotional enhancement hypothesis states that emotional stimuli attract more attention and encoding resources than do neutral stimuli, making the information easier to store; therefore, emotionally charged events tend to be remembered better than neutral events (Schweizer \& Dalgleish, 2016). Essentially, high levels of presence, engagement, and personal involvement (aspects IVR can offer) may contribute to memory retainment or learning.

In contrast, there are also theoretical reasons that VR could negatively impact children's learning. The emotional impairment hypothesis suggests that emotional content affects attention control and therefore the storage of information, thus interfering with memory performance (Garrison \& Schmeichel, 2018). Also, adding extraneous elements to a standard book (e.g., textures, sounds) can interfere with learning (Tare et al., 2010). For example, electronic books with many interactive features and sounds, or books with manipulatable features, such as pop-ups, tend to be a distraction from the text on the page, leading to worse story-content recall (Strouse et al., 2018). Thus, it is possible that interactivity, sounds, and manipulatable features in VR too could interfere with the learning process.

Additionally, a review on the use of IVR in education and training with adults found that while IVR can be effective for a number of purposes including the promotion of skill acquisition and fostering understanding of visuospatial information, VR technology does not always offer learning advantages over less immersive or traditional instruction. Moreover, in some instances VR can even be counterproductive to learning due to cybersickness, technological challenges, or because the immersive experience distracts from the learning task (Jensen \& Konradsen, 2018). For example, a study by Fernandes et al. (2016), found that presenting 3D sounds and a virtual rendering of user's own hands to an environment led to confusion and distraction which negatively impacted learning. 
Given the various reasons that an IVR experience could either enhance or distract from children's learning, this study aimed to investigate whether IVR technologies could generate more vivid emotions and increase memory achievement in children. To do so, we randomly assigned 6- to 7-year-old participants to experience an emotional story in one of three modalities: IVR, video, or a paper-based book. For each modality, we then assess the participant's memory performance and emotion identification. We hypothesized that (a) children's memory performance would be higher for the content presented via IVR, and (b) children would rate the emotional intensity felt by the protagonist of the story highest when viewing the story in the IVR medium.

\section{Material and Methods}

\subsection{Participants}

An initial pilot study with 10 participants was conducted to provide a formative evaluation of the procedures and instruments, followed by the formal experiment with 60 participants ( 30 female, 30 male) aged 6 and 7 years $(\mathrm{M}=7.0$ years, $\mathrm{SD}=0.6$ years $)$. Each participant was randomly assigned to one of three conditions: book $(n=20, M$ age $=6.9$ years, $S D=0.6$ years $)$, video $(n=20, M$ age $=6.8$ years, $S D=0.6$ years $)$, or IVR $(\mathrm{n}=20, \mathrm{M}$ age $=7.1$ years, $\mathrm{SD}=0.6$ years $)$. Two children were recruited but were not included in this data set for the following reasons: failure of recording equipment $(n=1)$ and procedural error $(n=1)$. Families were recruited from the university's greater metropolitan area. The study was approved by the Swinburne University of Technology Human Research Ethics Committee. Prior to beginning each session, oral consent (by the child) and written consent (by a parent) were obtained by an experimenter.

Results from a demographic survey completed by participants' parents indicated that previous IVR experience was uncommon for child participants (only $21 \%$ of parents indicated that their child had ever used an IVR headset). Among the participants that had previously used an IVR headset, the majority (60\%) had used it on a single occasion. The median range of participant annual household income was $\$ 100,000-\$ 150,000$ (AUD). Most parents reported having completed a tertiary degree or higher (67\%).

\subsection{Materials}

It is well-established that studies comparing learning outcomes from different mediums has inherent difficulties (Clark, 1983; Kozma, 1994). For example, without careful consideration in the planning stage of a study it is easy for differences in medium to become confounded with differences in instructional methods, content, and testing (Parong \& Mayer, 2018). The present study was designed to include as much experimental control as possible by ensuring that the three conditions included the same graphics (albeit in dynamic vs static form) and by complementing the book with some written representations of the sounds that appear in the film version (e.g., "*sigh*”, "wow!").

\subsubsection{Apparatus and software}

The material for each of the three conditions (book, video, IVR) was based on Henry, a free IVR film ( 8 min, $45 \mathrm{sec}$ ) created by Oculus story studio (2015) and narrated by Elijah Wood, which tells the story of Henry the Hedgehog, who is unhappy because he has no friends. The film was downloaded from the Oculus platform. The six basic elements of the story are:

- Element 1: Henry's spikes prevent him from making friends.

- Element 2: Henry has a birthday celebration with no friends.

- Element 3: Henry wishes for a friend.

- Element 4: Dog balloons magically come alive.

- Element 5: Balloons run away from Henry's hugs (because of his spikes).

- Element 6: The balloons return with a turtle who becomes Henry's friend. 
Children in the IVR condition watched the film with an IVR headset (see Figure 1), as it is intended to be watched. The specific IVR content used in this experiment was a 360-degree animated film that allowed the participant to stand within the animated environment and view Henry's house just by turning her/his head, but without interacting with the characters. Specifically, we used a DK2 Oculus Rift head-mounted display (HMD) with $960 \times 1080$ per-eye display resolution, 100 grades field of view, an internal tracking of $1,000 \mathrm{~Hz}$ and a positional tracking of $60 \mathrm{~Hz}$ of update rate, and a weight of 440 grams. The HMD received its video signal from a PC (Windows 7 SP1 64 bit, NVIDIA GTX970, Intel i5-4590).

Children in the video condition watched the same film on a 19" LCD computer monitor (instead of the HMD used in the IVR condition) (see Figure 1). This two-dimensional version of the film was created by screenrecording an experimenter viewing of the Henry IVR film using the HMD. The recording was made with slow and stable movements of the HMD to avoid any undesired camera vibrations and to avoid inducing any motion sickness. The focus remained on the key actions taking place throughout the story (i.e., focusing on Henry and the other characters rather than on unrelated aspects of the room to ensure that the participants did not miss important elements of the story).

For the book condition, a laminated, 30-page A4 book was created using screenshots from the two-dimensional video of Henry (see Figure 1). The film narration was transcribed into text printed underneath each of the pictures. A small amount of additional text was added to convey nonverbal information that was visually conveyed in the video presentation (e.g., "*sigh*”).

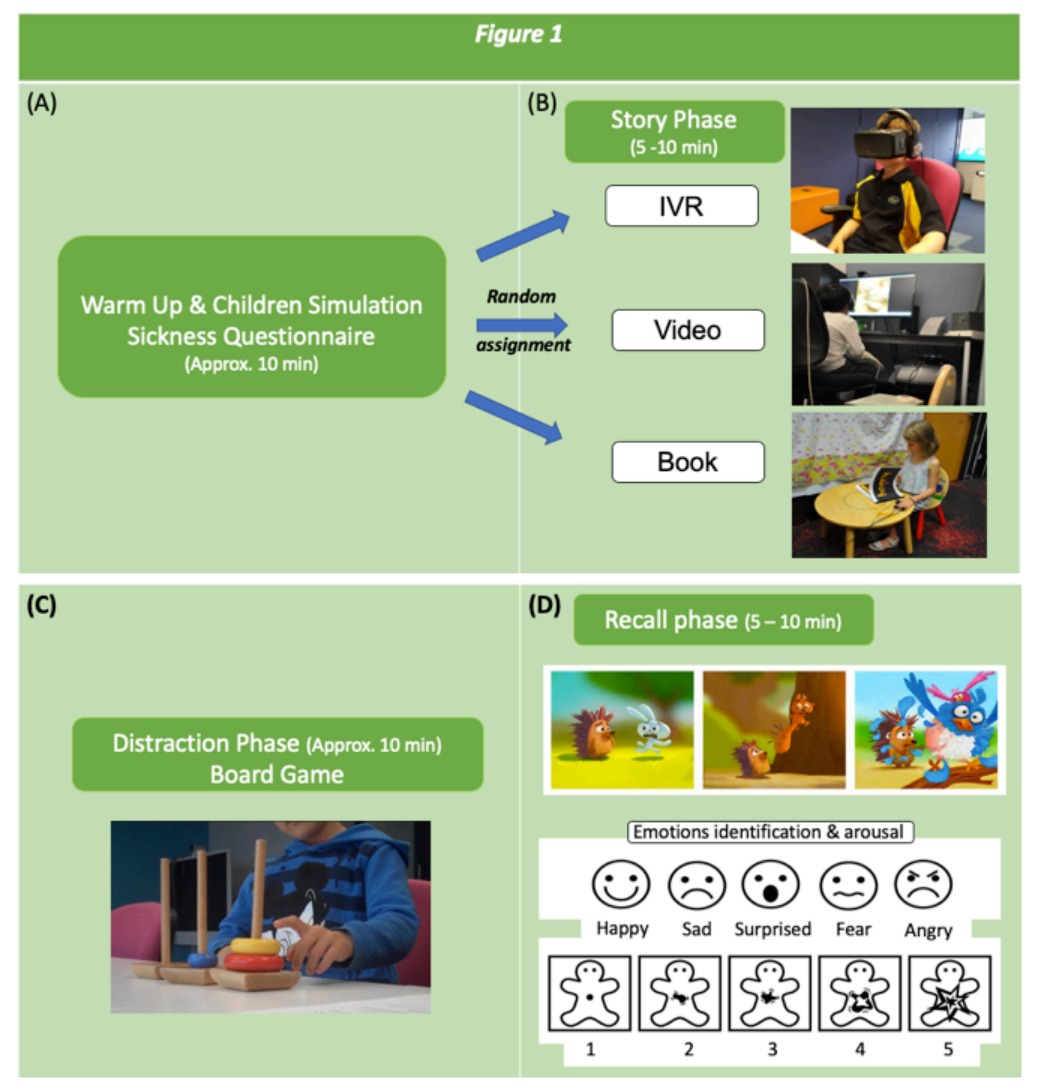

Figure 1. Illustration of study procedures. IVR = immersive virtual reality

\subsubsection{Assessment instruments}

Child Simulator Sickness Questionnaire (Hoeft et al., 2003): This seven-item instrument is designed to assesses specific pre-experiment symptoms of simulator sickness and collect information about the current physical condition and participant's experience with simulators. This questionnaire is an adaptation of the original Simulator Sickness Questionnaire for adults (Kennedy et al., 1993). The questionnaire poses seven 
questions related to potential feelings of sickness (e.g., "Do you have an upset stomach?", "Are you dizzy with your eyes closed?", etc.), and these questions are divided into three symptom categories: nausea, oculomotor, and disorientation. The questionnaire is scored by allocating each question a value of 0 to 2 based on the response; participants can answer no (score of 0 ), a little (score of 1), or a lot (score of 2). A score of 3 or more for any of the three symptom categories indicates the presence of simulator sickness symptoms.

Self-Assessment Manikin (SAM) (Bradley \& Lang, 1994): This nonverbal pictorial assessment technique directly measures the arousal associated with an affective reaction to stimuli. It consists of five different ratings (Likert-type scale), ranging from just a little to very much.

\subsection{Procedure}

The experiment consisted of four phases conducted in the following order: Warm-up Phase, Story Phase, Distraction Phase, and Recall Phase. Parents sat outside the testing room and completed the online demographic form and a media-use questionnaire while the child completed the study.

\subsubsection{Warm-up Phase (Approximately 10 min.)}

Previous research has demonstrated the importance of having a warm-up phase when conducting research with children (e.g., Allen et al., 2018). The warm-up allows the experimenter to establish rapport with a participant in order to reduce any anxiety that a child may experience due to being in an unfamiliar environment with stranger. A female researcher introduced herself to the child and played games with the child for approximately five minutes. After playing, the researcher asked the child to recount a story about something that happened to someone he or she knows that made that person feel sad or angry. This task ensured that children could tell a story that involves emotions in a logical order (100\% of participants succeeded). The researcher then carried out the Child Simulator Sickness Questionnaire with the child. As all children were able to recall a story, and none showed signs of sickness based on the questionnaire, all moved on to the Story Phase of the experiment.

\subsubsection{Story Phase (Approximately $5-10 \mathrm{~min}$.)}

Children were randomly assigned to one of the following three conditions: book, video, or IVR, as described above in the Materials section (see Figure 1). In the IVR condition, the researcher explained to the child that she or he would be watching a film about someone named Henry while wearing our VR goggles. The HMD was then fitted on the participant's head while seated in a swivel chair. Before the story commenced, the participant was asked to move her head in order locate the fireplace in the virtual environment. This was done to familiarize participants to the technology and to demonstrate that turning their heads enables them to see the entire scene (360-degrees). Following this, the experimenter explained to the child that different parts of Henry's house could be seen by turning one's head in different directions. The experimenter then began the IVR film. For children in the IVR condition, this phase lasted an additional 1-3 minutes so the headset could be fitted onto the head. The video condition was run in the same manner but displayed on the computer monitor rather than with the headset.

In the book condition, the researcher explained to the child that they would read a book about the story of Henry together. The researcher then placed the book in front of the child and read the story aloud. To avoid uncontrolled experimenter effects, a single researcher read the book the book all participants. The researcher was instructed to reflect the tone of the story, not read too fast and to vary the pace so she could introduce emphasis when the story required it. After completing each page, the researcher paused for 3 to 5 seconds before turning the page to ensure that the child had time to see all the images and printed material. 


\subsubsection{Distraction Phase (10 min.)}

The distraction phase consisted of 5 minutes of structured activity followed by 5 minutes of free play. The purpose of this phase was to create an interval between the stimulus and the memory-recall phase during which the participant is unlikely to rehearse the material presented in the story phase. The importance of a distraction phase is well-established in memory research with children (Riggins \& Rollins, 2015). A problem-solving game was selected as the structured activity to prevent participants from rehearsing the story during the delay. The child was instructed to try to solve the Tower of Hanoi problem which has previously been shown to be appropriate for children of this age (e.g., Huber et al., 2016; Tarasuik, Demaria \& Kaufman, 2017).

Free play consisted of the child playing with Lego while the researcher asked questions to establish a better rapport (e.g., "What's your favorite color?", "Do you play any sports?”).

\subsubsection{Recall Phase (Approximately $5-10 \mathrm{~min}$ )}

During this phase, the interviewer said to the child, "Please tell me the story of Henry. Tell me everything that you remember from the beginning to the end." While the child recounted the story, the researcher evaluated if the child recalled the six basic elements in the story. Each of the six elements received a binary score of 1 for correct recall and 0 for a miss. Participants were encouraged to continue when they seemed to stop their recounting by saying, "do you remember what happened after that?" but no clues were given to help retrieval.

To assess how children rated the emotional intensity felt by the protagonist of the story, the researcher presented each participant with emoji icons representing five emotions: sad, happy, angry, surprise, and fear. The children were asked, "Please tell me which of these feelings Henry felt in the story". Then, for each chosen emotion, the SAM (Bradley \& Lang, 1994) was used to assess the child's perception of the intensity of the emotion felt by Henry on a scale from 1 (just a little) to 5 ( $a$ lot).

\section{Results}

Our study was designed to assess our two main hypotheses that children in the IVR condition would recall more story content and would perceive Henry as feeling emotions more intensely, compared to the children in the other two conditions. Preliminary analyses revealed no significant main effects of child's sex and no interactions with sex on either of the key dependent measures (memory recall and emotions); therefore, sex was not included as a variable in subsequent analyses.

\subsection{Memory performance}

Across all ages, children recalled a median score of $4(\min =1, \max =6)$ scenes in the book condition, 5 ( $\min =1$, $\max =6)$ in the video condition, and $5(\min =4, \max =6)$ in the IVR condition. Although our initial analysis plan called for parametric analyses on memory scores, a Shapiro Wilk's test revealed that memory scores were not normally distributed, violating the normality assumption for an ANOVA $(p<.001)$. An examination of the data revealed that there were three general tiers of memory performance: perfect (scoring 6 out of 6 ), good (scoring 4 or 5 out of 6 ), and poor (scoring 0 to 3 out of 6 ). We therefore performed an ordinal logistic regression on the recoded memory scores according to these tiers using condition and age (in days) as predictors. Note, that the use of the recoded tiers ensured that there were at least five cases in more than $80 \%$ of the cells - a required assumption for the ordinal logistic regression.

The resulting regression on memory score tier revealed a main effect of condition, $\chi^{2}(2)=10.61, p=.005$; age $\chi^{2}(1)=5.43, p=.020$; and an age by condition interaction, $\chi^{2}(2)=7.68, p=.021$. Follow-up regressions on memory score tier for each condition separately indicated that there was a significant effect of age on memory score for children in the video condition only, $\chi^{2}(1)=9.50, p=.002$, with children in this condition performing increasingly better with age. 
To further probe the interaction involving age, we divided in children into two groups (6- and 7-year-olds) and evaluated the effects of condition for each age group separately.

\subsubsection{Six-year-old group}

A Kruskal-Wallis $\mathrm{H}$ test revealed a significant difference in the distribution of the memory score for the 6year-olds across condition $(H=6.66, p=0.036)$. A subsequent Mann-Whitney $\mathrm{U}$ test indicated that there was a significant difference in the distribution of the memory score between Video and IVR condition $(U=20.50$, $p=.019)$, as well as between Book and IVR condition $(U=12.00, p=.030)$. Overall, results illustrated in Figure 2 suggested that, among the 6-year-old children, those in the IVR condition best remembered the story content.

\subsubsection{Seven-year-old group}

We performed the same statistical analysis on the 7-year group data as we did for the 6-year-old group data to analyze memory score performance for this age group (Figure 2). The Kruskal-Wallis H test showed that the distribution of the memory score differed significantly across conditions, $H=12.66, p=0.002$. Mann-Whitney $\mathrm{U}$ test indicated that there was a significant difference in the distribution of the memory score between Video and Book condition $(U=6.50, p=.002)$, as well as between Book and IVR condition $(U=28.50, p=.009)$.

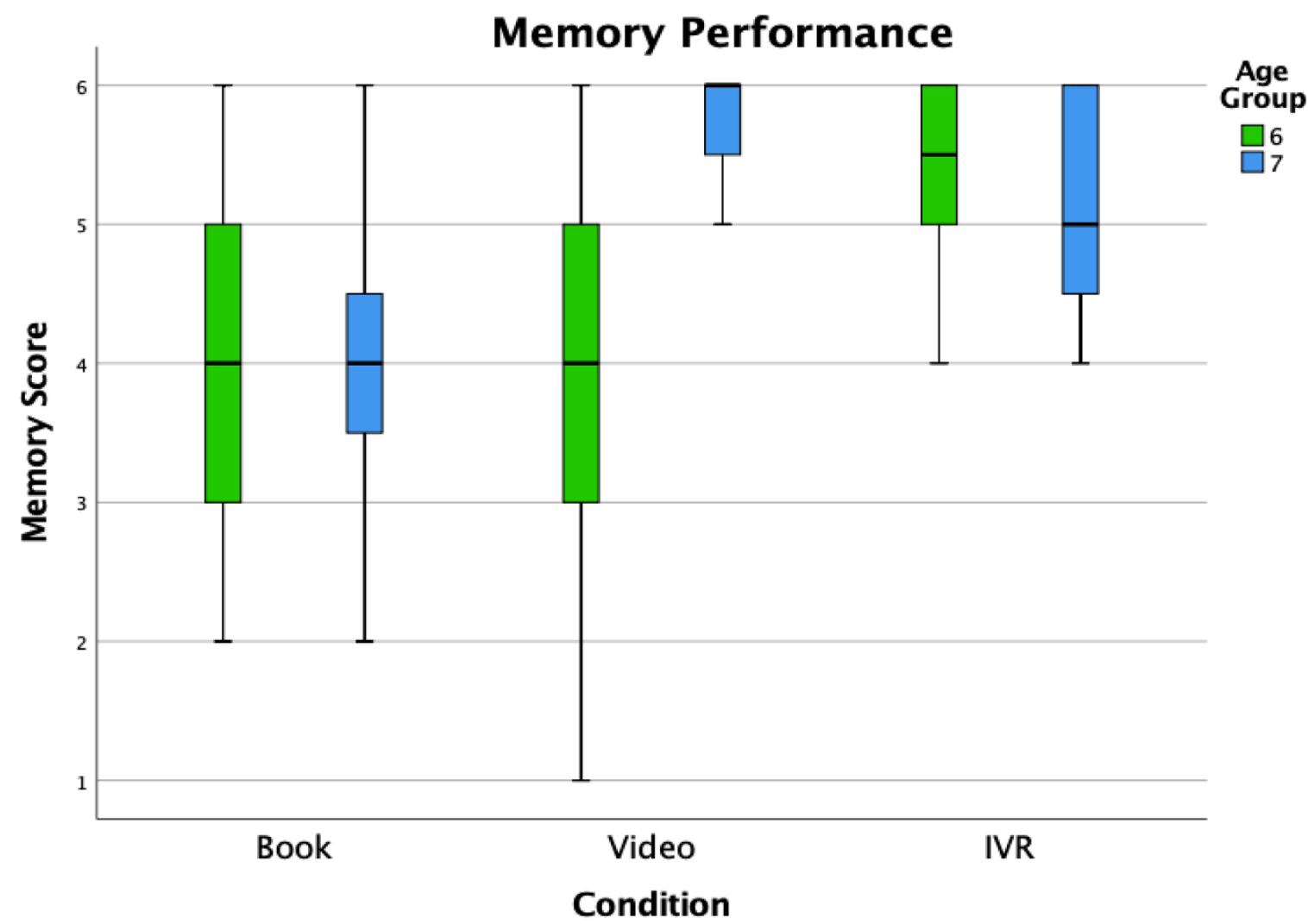

Figure 2. Boxplot of the distribution of memory scores for each condition and age (IVR $=$ immersive virtual reality).

\subsection{Ranking of Emotional Intensity}

We used a Scheirer-Ray-Hare test to examine the effect of condition and age (in days) on the children's ranking of emotional intensity felt by characters in the story. This analysis revealed a no statistically significant age $\times$ 
condition interaction, $H(2,60)=1.68, p=0.127$. Additionally, there was no significant condition effect $(H$ $(2,60)=1.98, p=0.163)$ and age effect $(H(18,60)=1.76, p=0.107)$ on the children's ranking of emotion.

The distribution of the ranking of the emotions was analyzed using a Kruskal-Wallis $\mathrm{H}$ test with condition (book, video, IVR) as the between-subject factor (Figure 3). The test revealed no significant difference in the distribution of the ranking of the emotions, $H=3.07, p=0.216$.

\subsection{Memory for Emotions Portrayed in Story}

We used a Kruskal-Wallis $\mathrm{H}$ test to examine the distribution of the total number of emotions that children attributed to Henry during the recall of the story, across condition and regardless of the intensity score attributed to these emotions. This analysis did not to reveal any significant difference in the distribution in the total number of emotions across condition $H=3.29, p=0.193$. Moreover, the majority of children independent of the condition they were in, were able to correctly identify the three primary emotions (sad, surprised and happy) displayed by the principal character (Henry) during the story.

\section{Ranking of Emotions}

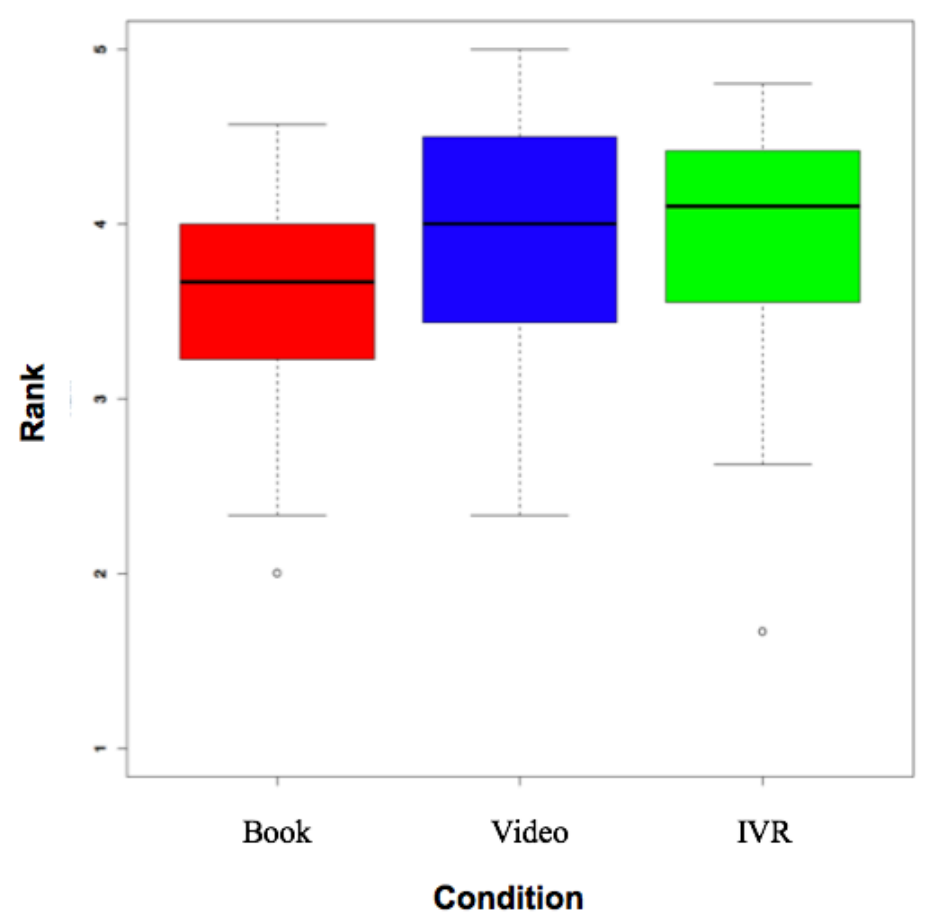

Figure 3. Boxplot of the distribution of the intensity of the emotions per condition (IVR $=$ immersive virtual reality).

\subsection{Child simulator sickness}

None of our participants attained a score of three or higher in the Child Simulator Sickness Questionnaire in any of the three symptom categories, indicating an absence of pre-experiment simulator sickness symptoms. Additionally, none of the participants reported any experience of motion sickness or any immediate side effects after using the VR headset.

\section{Discussion}

The purpose of this study was to determine whether IVR could increase memory achievement and generate more vivid emotions in children compared with video or hard-copy book modalities. Specifically, we set out 
to test two hypotheses related to the possibility that the immersive nature of the delivery could minimize distractions during encoding and provide a more engaging experience. First, we hypothesized that children's memory performance would be higher for the content presented via IVR. Second, we hypothesized that children would rate the emotional intensity felt by the protagonist of the story highest when viewing the story in the IVR medium compared with the other two conditions. Each hypothesis is discussed in turn below.

\subsection{Did story medium affect memory of story content?}

Our evaluation of memory for story content indicated that children in the IVR condition performed better in the memory-recall task than the children in the video and book conditions. A further breakdown of the memory recall data showed that 7-year-old participants in the digital-media conditions (IVR and video) performed better in the memory-recall task than the participants in the book condition. However, for the 6-year-old group, the results showed that participants in the IVR condition had better scores in the memory task than the participants in the video and book conditions.

One possible explanation for why children remembered the story better when presented through IVR could be the ability of this technology to stimulate the senses of the participant and present the information in a more realistic manner (Bell et al., 1995). Such realism may be less cognitively demanding because the viewer is more directly immersed in the story and less active imagination of the world is required (Shibli \& West, 2018). Sense of presence has been defined as the perceptual illusion of being there, and previous work with children and adults has shown that high levels of sense of presence and emotional involvement can facilitate later recall (Mantovani \& Castelnuovo, 2003). Furthermore, VR technology can combine visually immersive spatial representations of data with our vestibular and proprioceptive senses, which offers a distinctive spatial mnemonic that also assists in the memory recall (Krokos et al., 2019).

Children's memory may also have benefitted from the IVR condition because of the greater level of engagement it affords (Mantovani \& Castelnuovo, 2003). In a study with adults in which the effects of IVR on performance, emotion, and engagement were compared with effects of textbook and video learning, participants found content presented through IVR more engaging and easier to remember than the participants receiving the same content via textbook or video (Allcoat \& Adrian, 2018). Moreover, engagement can be fostered with virtual environments (Dalgarno \& Lee, 2010) and an important aspect of learning (i.e., the greater the engagement, the greater the learning) (McMahan, 2003; Pintrich, 2003). Therefore, the content presented through IVR may have been more engaging and attractive for the children, leading to higher memory recall.

Despite the advantages presented above about the potential of IVR as a memory aid, other studies point out the possible drawbacks of this technology. For example, previous research has suggested that adding interactive features or entertaining content to a story can be distracting and interfere with encoding of the story content, resulting in worse memory recall (Chiong et al., 2012; De Jong \& Bus, 2002; Hirsh-Pasek et al., 2015; Tare et al., 2010). Indeed, as reported by Daniaeu, Guillo and Dore (2017), IVR implementations should include elements to guide attention to the key content to avoid users becoming distracted by less important aspects of the VR environment. Our results suggest that the narrative itself served this purpose as children's memory of the content was not negatively affected by the possibility of viewing story-irrelevant spaces in the IVR condition. Instead, our results are more consistent with previous work showing the comprehension and retention benefits when interactivity is embedded thoughtfully into the story, as the features can increase attention and engagement with the content (Etta \& Kirkorian, 2018; Lauricella et al., 2014).

\subsection{Did IVR lead children to attribute stronger emotions to the protagonist?}

The IVR medium is routinely suggested to potentially generate more vivid visceral experiences for its users due to its qualities of immersion and sense of presence and its ability to allow the users to experience any situation from multiple perspectives (Herrera et al., 2018; Ravaja et al., 2005). Thus, we hypothesized that our 
participants that experienced IVR would attribute stronger emotions to Henry during the story. However, our results did not reveal a significant difference in the ranking of emotional arousal for the participants using IVR compared with the participants watching (video condition) or reading (book condition) the story.

Indeed, children in all conditions were able to correctly identify the emotions and reasonably rank their arousal according to the story. This is not surprising in that 6- and 7-year-olds have the capacity to identify and measure the level of emotions of a character in a story (Saarni, 2011). During early and middle childhood, children become more aware of their own and other's feelings and more capable of understanding emotion "scripts." This ability to understand and recognize emotions may be developed well enough such that children in all conditions were able to respond appropriately, making it more difficult to demonstrate a possible IVR content effect.

Moreover, our focus was on basic emotions (e.g., sadness, happiness) that are easy to recognize in the context of a good story (Henry is an Emmy-winning story), rather than more subtle emotions (e.g., fear, disgust, surprise). The focus on basic emotions may have made measuring an effect of story medium more difficult. In a study by Lawrence et al. (2016), in which the ability of children (aged 6-11 years) and adolescents (aged 11-16 years) to recognize emotions was evaluated through an emotion recognition test, the authors found that happiness, sadness, and anger were recognized by 6-year-olds almost as well as they were by the adolescents; recognizing more-complex emotions seems to be more difficult for younger children, but this ability improves throughout childhood and adolescence.

\subsection{Limitations and future directions}

The following limitations should be taken into account when interpreting the results of this experimental study. First, the interpretation of specific outcomes is based on cognitive load and engagement factors; but these factors were not explicitly measured. Therefore, there is scope for future studies to build on this work by including a robust measure of cognitive load experienced by children when immersed in the VR and non-VR environments. Regarding engagement, although we did not measure this construct directly, there exist studies of adults showing high levels of engagement during training when using VR technology (e.g., Diego-Mas et al., 2020) and recent studies carried out in our lab have demonstrated that children show greater levels of engagement and enjoyment when learning through IVR compared to regular teaching tools (Araiza-Alba et al., 2020b, 2020c). A second limitation relates to possible confounding factors across the conditions. Specifically, "Henry VR" is a film design for VR use, and although we put considerable thought and effort into creating understandable and comparable non-VR versions of the Henry story, it is possible that the results may be different with a story that was designed to be presented across numerous platforms. Finally, it is possible that our failure to reveal media condition effects on emotion identification may be due to ceiling effects based on the ease of identifying basic emotions. A better approach for future studies may be to examine more complex emotions (e.g., fear, disgust, surprise).

Our study provides a glimpse into what may lie ahead in the field of IVR as a tool to enhance children's memory. Therefore, a next step is for research to identify and characterize what elements of the virtual environment are most effective in eliciting greater information recall. At present, we have studied the effect of an immersive experience that does not allow active participation or interaction with the virtual environment. Participants were only allowed to freely rotate their viewpoint to experience the content through the HMD. A valuable study would be to examine how the addition of interaction and active participation impact memory recall (Allcoat \& Adrian, 2018). 


\section{Conclusion}

Overall, IVR does seem to be a useful at improving memory encoding for younger children, perhaps by stimulating their senses and presenting information in a more realistic manner-providing them with a global frame of reference and placing less cognitive load on children's minds giving them the opportunity to focus on and remember the information presented through IVR. These exciting results suggest that IVR can be beneficial for assisting memory encoding and offer insight into how children's memory can be enhanced by this technology, not only as a potential memory aid but also as a possible means to help children learn. Moreover, our finding that children generally had positive experiences with IVR in conjunction with recent developmental VR studies of child perception (e.g., (Negen et al., 2018; Valori et al., 2020)) suggests that this technology can join others such as touchscreens and robots (e.g., (Martin et al., 2019, 2020)) in the growing technological pantheon of tools to probe important aspects of children's developing minds.

\section{Acknowledgements}

We thank the many parents and children who gave their time to participate in this research. We also thank the Swinburne Babylab interns for their help carrying out this research. Paola Araiza-Alba expresses her gratitude to the Mexican National Council for Science and Technology (CONACYT) and to Swinburne University of Technology for the research scholarship funding that supported this research.

\section{Conflict of Interest Statement}

The authors declare no conflicts of interest.

\section{References}

Allcoat D. and Adrian M., 2018. Learning in virtual reality: Effects on performance, emotion and engagement. Research in Learning Technology, 26(1063519), 1-13. doi:10.25304/rlt.v26.2140

Allen M., Perry C., Kaufman J., 2018. Toddlers prefer to help familiar people. Journal of Experimental Child Psychology, 174, 90-102. doi:10.1016/j.jecp.2018.05.009

Araiza-Alba P., Keane T., Beaudry J.L., Kaufman J., 2020a. Immersive Virtual Reality Implementations in Developmental Psychology. International Journal of Virtual Reality, 20(2). doi:10.20870/IJVR.2020.20.2.3094

Araiza-Alba P., Keane T., Chen W.S., Kaufman J., 2020b. Immersive virtual reality as a tool to learn problem-solving skills. PsyArXiv. doi:10.31234/osf.io/q857x

Araiza-Alba P., Keane T., Matthews B., Simpson K., Strugnell G., Chen W.S., Kaufman J., 2020c. The potential of 360-degree VR videos to teach water-safety skills to children. Computers \& Education, 163, 104096. doi:10.1016/j.compedu.2020.104096

Bell J.T., Fogler H.S., Arbor A., 1995. The Investigation and Application of Virtual Reality as an Educational Tool. 2513, 1-11. http://www.cs.uic.edu/ jbell/Professional/Papers/aseepap2.pdf

Bhagat K.K., Liou W.K., Chang C.Y., 2016. A cost-effective interactive 3D virtual reality system applied to military live firing training. Virtual Reality, 20(2), 127-140. doi:10.1007/s10055-016-0284-x

Bradley M.M. and Lang P.J., 1994. Measuring emotion: The self-assessment manikin and the semantic differential. Journal of Behavior Therapy and Experimental Psychiatry, 25(1), 49-59. doi:10.1016/0005-7916(94)90063-9

Bricken M., 1991. Virtual reality learning environments: potentials and challenges. ACM SIGGRAPH Computer Graphics, 25(3), 178-184. doi:10.1145/126640.126657

Bryant L., Hemsley B., Bailey B., Bluff A., Nguyen V., Stubbs P., Barnett D., Jacobs C., Lucas C., Power E., 2020. Opportunities for the Implementation of Immersive Virtual Reality in Rehabilitation.

Proceedings of the 53rd Hawaii International Conference on System Sciences. doi: $10.24251 /$ hicss.2020.437

Chen Y.L., 2016. The Effects of Virtual Reality Learning Environment on Student Cognitive and Linguistic 
Development. Asia-Pacific Education Researcher, 25(4), 637-646. doi:10.1007/s40299-016-0293-2

Chiong C., Ree J., Takeuchi L., Erickson I., 2012. Print Books vs. E-books Comparing parent-child coreading on print, basic, and enhanced e-book platforms A Cooney Center QuickReport by. http://www.joanganzcooneycenter.org/wp-content/uploads/2012/07/jgcc_ebooks_quickreport.pdf

Clark R.E., 1983. Reconsidering Research on Learning from Media. Review of Educational Research, 53(4), 445-459. doi:10.3102/00346543053004445

Dalgarno B. and Lee M.J.W., 2010. What are the learning affordances of 3-D virtual environments? British Journal of Educational Technology, 41(1), 10-32. doi:10.1111/j.1467-8535.2009.01038.x

De Jong M.T. and Bus A.G., 2002. Quality of Book-Reading Matters for Emergent Readers: An Experiment With the Same Book in a Regular or Electronic Format. doi:10.1037/0022-0663.94.1.145

Dibbets P. and Schulte-Ostermann M.A., 2015. Virtual reality, real emotions: a novel analogue for the assessment of risk factors of post-traumatic stress disorder. Frontiers in Psychology, 6(May), 1-8. doi:10.3389/fpsyg.2015.00681

Diego-Mas J.A., Alcaide-Marzal J., Poveda-Bautista R., 2020. Effects of Using Immersive Media on the Effectiveness of Training to Prevent Ergonomics Risks. International Journal of Environmental Research and Public Health, 17(7), 2592. doi:10.3390/ijerph17072592

Etta R.A. and Kirkorian H.L., 2018. Children's learning from interactive eBooks: Simple irrelevant features are not necessarily worse than relevant ones. Frontiers in Psychology, 9(JAN), 2733. doi:10.3389/fpsyg.2018.02733

Garrison K.E. and Schmeichel B.J., 2018. Effects of emotional content on working memory capacity. Cognition and Emotion, 1-8. doi:10.1080/02699931.2018.1438989

Gathercole S.E., 1998. The development of memory. In Journal of Child Psychology and Psychiatry and Allied Disciplines (Vol. 39, Issue 1, pp. 3-27). doi:10.1017/S0021963097001753

Harman J., Brown R., Johnson D., 2017. Improved Memory Elicitation in Virtual Reality: New Experimental Results and Insights. Lecture Notes in Computer Science (Including Subseries Lecture Notes in Artificial Intelligence and Lecture Notes in Bioinformatics), 10514 LNCS, 128-146. doi:10.1007/978-3319-67684-5_9

“Henry” Oculus Story Studio. (2015). Oculus Story Studio. https://www.oculus.com/storystudio/films/henry/?locale=en_US

Herrera F., Bailenson J., Weisz E., Ogle E., Zak J., 2018. Building long-term empathy: A large-scale comparison of traditional and virtual reality perspective-taking. PLOS ONE, 13(10), e0204494. doi:10.1371/journal.pone.0204494

Hirsh-Pasek K., Zosh J.M., Golinkoff R.M., Gray J.H., Robb M.B., Kaufman J., 2015. Putting Education in "Educational" Apps: Lessons From the Science of Learning. In Psychological Science in the Public Interest, 16(1), 3-34. doi:10.1177/1529100615569721

Hoeft R.M., Vogel J., Bowers C.A., 2003. Kids Get Sick Too: A Proposed Child Simulator Sickness Questionnaire. Proceedings of the Human Factors and Ergonomics Society Annual Meeting, 47(20), 2137-2141. doi:10.1177/154193120304702013

Huang H.-M., Rauch U., Liaw S.S., 2010. Investigating learners' attitudes toward virtual reality learning environments: Based on a constructivist approach. Computers \& Education, 55(3), 1171-1182. doi:10.1016/j.compedu.2010.05.014

Huber B., Highfield K., Kaufman J., 2018. Detailing the digital experience: Parent reports of children's media use in the home learning environment. British Journal of Educational Technology, 49(5), 821833. doi:10.1111/bjet.12667

Huber B., Meyer D., Kaufman J., 2020. Young children's contingent interactions with a touchscreen influence their memory for spatial and narrative content. Media Psychology, 23(4), 552-578. doi:10.1080/15213269.2019.1611451

Huber B., Tarasuik J., Antoniou M.N., Garrett C., Bowe S.J., Kaufman J., 2016. Young children's transfer of learning from a touchscreen device. Computers in Human Behavior, 56, 56-64. 
doi:10.1016/j.chb.2015.11.010

Ibáñez M.B., Jesús G.J., Sergio G., David M., Morillo D., Delgado-Kloos C., 2011. Design and Implementation of a 3D Multi-User Virtual World for Language Learning. Journal of Educational Technology \& Society, 14(4), 2-10. http://www.jstor.org/stable/jeductechsoci.14.4.2

Jablon J.R. and Wilkinson M., 2006. Engagement Strategies to Facilitate Children's Learning and Success. Beyond the Journal, March, 1-5.

https://www.academia.edu/15720795/Using_Engagement_Strategies_to_Facilitate_Childrens_Learning _and_Success

Jensen L. and Konradsen F., 2018. A review of the use of virtual reality head-mounted displays in education and training. Education and Information Technologies, 23(4), 1515-1529. doi:10.1007/s10639-0179676-0

Ke F. and Im T., 2013. Virtual-Reality-Based Social Interaction Training for Children with HighFunctioning Autism. The Journal of Educational Research, 106(6), 441-461. doi:10.1080/00220671.2013.832999

Kennedy R.S., Lane N.E., Berbaum K.S., Lilienthal M.G., 1993. Simulator Sickness Questionnaire: An Enhanced Method for Quantifying Simulator Sickness. The International Journal of Aviation Psychology, 3(3), 203-220. doi:10.1207/s15327108ijap0303_3

Kipping B., Rodger S., Miller K., Kimble R.M., 2012. Virtual reality for acute pain reduction in adolescents undergoing burn wound care: A prospective randomized controlled trial. In Burns (Vol. 38, Issue 5, pp. 650-657). doi:10.1016/j.burns.2011.11.010

Kozma R., 1994. The influence of media on learning: The debate continues. School Library Media Quarterly, 22(4), 1-13.

Krokos E., Plaisant C., Varshney A., 2019. Virtual memory palaces: immersion aids recall. Virtual Reality, 0123456789, 1-15. doi:10.1007/s10055-018-0346-3

Lauricella A.R., Barr R., Calvert S.L., 2014. Parent-child interactions during traditional and computer storybook reading for children's comprehension: Implications for electronic storybook design. International Journal of Child-Computer Interaction, 2(1), 17-25. doi:10.1016/j.ijcci.2014.07.001

Lawrence K., Campbell R., Skuse D.H., 2016. Can Children See Emotions in Faces? Frontiers for Young Minds, 4. doi:10.3389/frym.2016.00015

León I., Cimadevilla J.M., Tascón L., 2014. Developmental Gender Differences in Children in a Virtual Spatial Memory Task. Neuropsychology, 28(4), 485-495. doi:10.1037/neu0000054

Loureiro S.M.C., Guerreiro J., Eloy S., Langaro D., Panchapakesan P., 2019. Understanding the use of Virtual Reality in Marketing: A text mining-based review. Journal of Business Research, 100, 514 530. doi:10.1016/j.jbusres.2018.10.055

Mania K., Troscianko T., Hawkes R., Chalmers A., 2003. Fidelity metrics for virtual environment simulations based on spatial memory awareness states. Presence: Teleoperators and Virtual Environments, 12(3), 296-310. doi:10.1162/105474603765879549

Mantovani F. and Castelnuovo G., 2003. Sense of Presence in Virtual Training: Enhancing Skills Acquisition and Transfer of Knowledge through Learning Experience in Virtual Environments. Being There: Concepts, Effects and Measurement of User Presence in Synthetic Environments, January, 168181.

Maria R., Johnson A., Moher T., Leigh J., Vasilakis C., Barnes C., 1999. Learning and building together in an immersive virtual world. Presence: Teleoperators and Virtual Environments, 8(3), 247-263. doi:10.1162/105474699566215

Martin D.U., MacIntyre M.I., Perry C., Clift G., Pedell S., Kaufman J., 2020. Young Children's Indiscriminate Helping Behavior Toward a Humanoid Robot. Frontiers in Psychology, 11, 239. doi:10.3389/fpsyg. 2020.00239

Martin D.U., Perry C., MacIntyre M.I., Varcoe L., Pedell S., Kaufman J., 2019. Investigating the nature of children's altruism using a social humanoid robot. Computers in Human Behavior, 104, 106149. 
doi:10.1016/j.chb.2019.09.025

Mayer R.E., 2003. The promise of multimedia learning: Using the same instructional design methods across different media. Learning and Instruction, 13(2), 125-139. doi:10.1016/S0959-47520200016-6

McMahan A., 2003. Immersion, Engagement and Presence: A Method for Analyzing 3-D Video Games | Alison McMahan, Writer. In The Video Game, Theory Reader (pp. 67-86). Routledge. doi:10.4324/9780203700457-10

Negen J., Heywood-Everett E., Roome H.E., Nardini M., 2018. Development of allocentric spatial recall from new viewpoints in virtual reality. Developmental Science, 21(1), 1-29. doi:10.1111/desc. 12496

Papanastasiou G., Drigas A., Skianis C., Lytras M., Papanastasiou E., 2018. Virtual and augmented reality effects on K-12, higher and tertiary education students' twenty-first century skills. Virtual Reality, 23(4), 425-436. doi:10.1007/s10055-018-0363-2

Parong J. and Mayer R.E., 2018. Learning science in immersive virtual reality. Journal of Educational Psychology, 110(6), 785-797. doi:10.1037/edu0000241

Pausch R., Proffitt D., Williams G., 1997. Quantifying immersion in virtual reality. Proceedings of the 24th Annual Conference on Computer Graphics and Interactive Techniques, SIGGRAPH 1997, 13-18. doi:10.1145/258734.258744

Pintrich P.R., 2003. A Motivational Science Perspective on the Role of Student Motivation in Learning and Teaching Contexts. Journal of Educational Psychology, 95(4), 667-686. doi:10.1037/00220663.95.4.667

Ravaja N., Salminen M., Holopainen J., Saari T., Laarni J., Järvinen A., 2005. Emotional response patterns and sense of presence during video games. 339-347. doi:10.1145/1028014.1028068

Riggins T. and Rollins L., 2015. Developmental Differences in Memory During Early Childhood: Insights From Event-Related Potentials. Child Development, 86(3), 889-902. doi:10.1111/cdev.12351

Saarni C., 2011. Emotional development in childhood. Encyclopedia on Early Childhood Development. http://www.child-encyclopedia.com/sites/default/files/textes-experts/en/638/emotional-development-inchildhood.pdf

Schweizer S. and Dalgleish T., 2016. The impact of affective contexts on working memory capacity in healthy populations and in individuals with PTSD. Emotion, 16(1), 16-23. doi:10.1037/emo0000072

Shibli D. and West R., 2018. Cognitive Load Theory and its Application in the Classroom. Journal of the Chartered College of Teaching, 2(Science of Learning). https://impact.chartered.college/article/shiblicognitive-load-theory-classroom/

Slater M. and Wilbur S., 199. A framework for immersive virtual environments (FIVE): Speculations on the role of presence in virtual environments. Presence: Teleoperators and Virtual Environments, 6(6), 603-616. doi:10.1162/pres.1997.6.6.603

Strouse G.A., Nyhout A., Ganea P.A., 2018. The role of book features in young children's transfer of information from picture books to real-world contexts. In Frontiers in Psychology (Vol. 9, Issue FEB, pp. 1-14). doi:10.3389/fpsyg.2018.00050

Tarasuik J., Demaria A., Kaufman J., 2017. Transfer of Problem Solving Skills from Touchscreen to 3D Model by 3- to 6-Year-Olds. Frontiers in Psychology, 8(SEP), 1586. doi:10.3389/fpsyg.2017.01586

Tarasuik J., Galligan R., Kaufman J., 2011. Almost being there: Video communication with young children. PLoS ONE, 6(2), e17129. doi:10.1371/journal.pone.0017129

Tarasuik J., Galligan R., Kaufman J., 2013. Seeing is believing but is hearing? Comparing audio and video communication for young children. Frontiers in Psychology, 4(FEB), 64. doi:10.3389/fpsyg.2013.00064

Tarasuik J. and Kaufman J., 2017. When and why parents involve young children in video communication. Journal of Children and Media, 11(1), 88-106. doi:10.1080/17482798.2016.1233124

Tare M., Chiong C., Ganea P., DeLoache J., 2010. Less is more: How manipulative features affect children's learning from picture books. Journal of Applied Developmental Psychology, 31(5), 395-400. doi:10.1016/j.appdev.2010.06.005 
Valori I., McKenna-Plumley P.E., Bayramova R., Zandonella Callegher C., Altoè G., Farroni T., 2020. Proprioceptive accuracy in Immersive Virtual Reality: A developmental perspective. PLOS ONE, 15(1), e0222253. doi:10.1371/journal.pone.0222253

Wang F., Gao C., Kaufman J., Tong Y., Chen J., 2021. Watching versus touching: The effectiveness of a touchscreen app to teach children to tell time. Computers and Education, 160, 104021. doi:10.1016/j.compedu.2020.104021

Wilson C.J., Soranzo A., Sheffield S., 2015. The Use of Virtual Reality in Psychology : A Case Study in Visual Perception. Computational and Mathematical Methods in Medicine, 2015. doi:10.1155/2015/151702

Winn W., 2010. A Conceptual Basis for Educational Applications http://www.hitl.washington.edu/publications/r-93-9/|. Technical Report TR-93-9, Human Interface Technology Laboratory-University of Washington., 1-13.

http://www.hitl.washington.edu/research/learning_center/winn/winn-paper.html 